\title{
Intramolecular, Visible Light-Mediated Aza Paternò-Büchi Reactions of Unactivated Alkenes
}

\author{
Dominique E. Blackmun, Stephen A. Chamness, and Corinna S. Schindler.
}

University of Michigan, Department of Chemistry, Willard-Henry-Dow Laboratory, 930 N University Ave., Ann Arbor, MI 48109

Supporting Information Placeholder

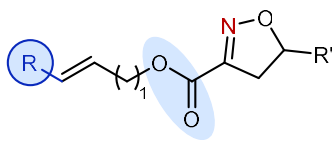

unactivated alkenes

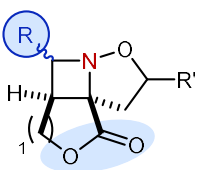

complex highly saturated scaffold

\begin{abstract}
Azetidines are of particular interest in medicinal chemistry for their favorable properties, including increased resistance to oxidative metabolism and lower lipophilicity. The recent development of [2+2] reactions has significantly benefitted the previously limited methods for azetidine synthesis, but access to more complex architectures still requires further development. Herein we report a visible-light enabled intramolecular [2+2] cycloaddition to access tricyclic azetidines with 3D complex structures and high levels of saturation.
\end{abstract}

Incorporation of highly substituted, three-dimensional scaffolds into target structures is an essential strategy in medicinal chemistry to facilitate the development of future pharmaceuticals. ${ }^{1}$ Current discovery efforts are shifting towards the inclusion of more saturated, complex scaffolds to increase the likelihood of identifying promising lead structures.. ${ }^{1}$ Benefits from higher saturation and higher complexity range from increased solubility to greater specificity, and lower toxicity, desirable qualities for a drug. ${ }^{2}$ Azetidines are 4-membered nitrogen-containing heterocycles that recently garnered a lot of attention as medicinally desirable scaffolds in the quest for new lead compounds in drug design and development. ${ }^{3}$ Specifically, azetidines exhibit favorable pharmacokinetic properties and biological activities including decreased lipophilicity, high polarity, and rigidity, in addition to facilitating the rapid build-up of molecular complexity. ${ }^{1,2,4-9}$ Implementation of azetidines as alternatives for 5- and 6-membered nitrogen-containing heterocycles in current potential therapeutic candidates is known to improve their metabolic stability by enhancing their overall structural rigidity, resulting in enhanced resistance to oxidative metabolism. ${ }^{3-6}$ Furthermore, these compounds often exhibit lower lipophilicity and higher polarity, which represent additional important contributions to enhance the efficacy of a compound..$^{5}$ For example, $5-\mathrm{HT}_{2 \mathrm{C}}$ agonists, $\mathbf{1}$ and $\mathbf{2}$ identified by Pfizer, differ only in the ring size of the $\mathrm{N}$-heterocycle, yet the azetidine results in a more potent compound with an $\mathrm{EC}_{50}$ of $12 \mathrm{nM}$ compared to $273 \mathrm{nM}$ (Fig. $1 \mathrm{~A}){ }^{4}$
However, despite these desirable characteristics, the incorporation of azetidines in current pharmaceutical lead compounds remains limited. Importantly, less than $1 \%$ of all nitrogen-heterocycles included in current FDA-approved pharmaceuticals are functionalized azetidines. ${ }^{10}$ This divide is due to a dearth in efficient synthetic methods to access these desirable 4-membered ring nitrogen-containing heterocycles. ${ }^{11-15}$ Among the synthetic strategies most often

Figure 1. A. Azetidines as important scaffolds for current drug design and development. B. Currently available synthetic strategies to access functionalized azetidines.

A. Beneficial Effects of Azetidine Scaffolds on Pharmacokinetics

metabolically at risk

metabolically robust
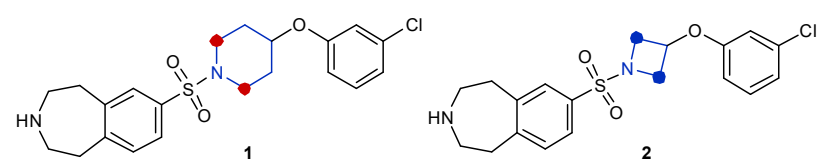

$\mathrm{EC}_{50}(\mathrm{nM})=273$

$\mathrm{EC}_{50}(\mathrm{nM})=12$

advantages of azetidines:

- rigid scaffold - high polarity - low lipophilicity - increased metabolic stability

B. Select Synthetic Strategies towards Azetidines

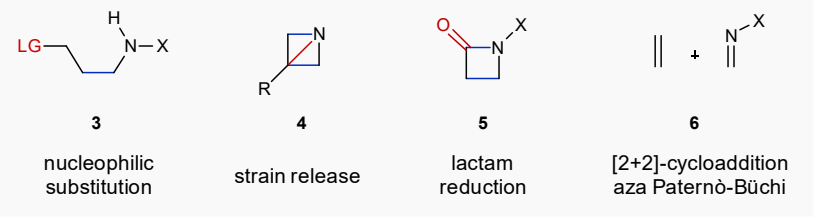


Figure 2. A. Intramolecular methodology using excited alkenes. B. Intermolecular methodology using excited oximes and unactivated alkenes. C. Novel intramolecular methodology using excited oximes and unactivated alkenes.

A. Previously: IntramolecularAza Paternò-Büchi Reactions; Styrene/Diene Activation
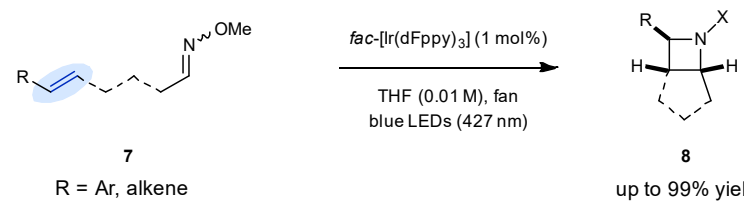

8

up to $99 \%$ yield

B. Previously: IntermolecularAza Paternò-Büchi Reactions; Activation of the Isoxazoline
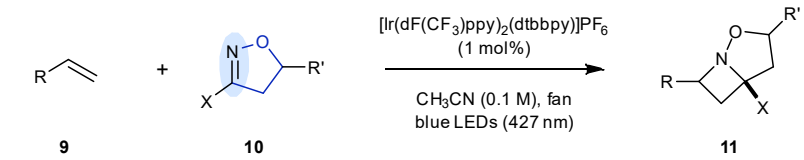

unactivated $\mathrm{X}=\mathrm{CO}_{2} \mathrm{R}, \mathrm{CN}$ blue LEDs $(427 \mathrm{~nm})$

11 up to $99 \%$ yield

C. This Work: Intramolecular Aza Paternò-Büchi Reactions: Isoxazoline Activation

$$
\text { unactivated alkenes }
$$

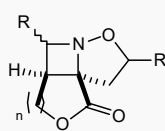

13

complex azetidine scaffolds up to $87 \%$ yield applied are nucleophilic substitutions (3),13,14 strain release of azabicyclobutanes (4), ${ }^{13-15}$ and the reduction of lactams (5, Fig. 1B). ${ }^{13,14}$ However, all of these approaches require the synthesis of specific precursors and are prone to limitations in scope. Arguably, the most efficient and direct strategy towards functionalized azetidines relies on [2+2]-cycloaddition reactions between imines and alkenes, or an aza Paternò-Büchi reaction (6). Select efficient procedures for these aza Paternò-Büchi reactions have recently been developed, relying on visible light-mediated energy transfer (Fig. 2). These methods harness the triplet energy excited states of either alkenes or oximes, generated upon energy transfer from the triplet excited states of suitable photocatalysts. ${ }^{16-19}$ Following this approach, intramolecular aza Paternò-Büchi reactions proceed upon accessing the triplet excited states of activated alkenes (7) to undergo the desired [2+2]-cycloaddition with pendant oximes in up to 99\% yield to form azetidines (8) (Fig. 2A). ${ }^{16}$ An intermolecular variant of this transformation was subsequently developed that relies on unactivated alkenes 9 and the triplet excited states of cyclic oximes $\mathbf{1 0}$ to promote the formation of azetidines 11 in up to $99 \%$ yield (Fig. 2B). ${ }^{17}$ Herein we report the development of an intramolecular aza PaternòBüchi reaction of unactivated alkenes by relying on the design principle of oxime excitation to access complex azetidine scaffolds. Specifically, cyclic oximes react with the unactivated alkene moieties in $\mathbf{1 2}$ to result in the formation of tricyclic azetidines $\mathbf{1 3}$ to access substrates with opposite substitution patterns in comparison to the previous intermolecular approach. Notably, both metal-based and organic photocatalysts are shown to promote the desired transformation in yields up to $87 \%$ and $41 \%$ respectively.
To identify optimal conditions for intramolecular aza Paternò-Büchi reactions of unactivated alkenes, we evaluated a range of photocatalysts varying in triplet energies upon irradiation of oxime $\mathbf{1 4}$ with blue LED lights (Table 1). Specifically, conversion of oxime $\mathbf{1 4}$ with catalytic amounts of photocatalysts displaying a high triplet energy around 60 $\mathrm{kcal} \mathrm{mol}^{-1}$ including $\operatorname{Ir}\left(\mathrm{dF}(\mathrm{Me})\right.$ ppy) ${ }_{2}$ (dtbbpy)] and $\mathrm{Ir} 1 \cdot \mathrm{PF}_{6}$ resulted in the formation of azetidine 15 in $73 \%$ and $78 \%$ yield, respectively (entries 1-2, Table 1). Under otherwise identical reaction conditions, conversion of oxime 14 with Ir2 gave rise to azetidine 15 with increased yields of $90 \%$ (entry 3, Table 1). Using a catalyst with a lower triplet energy of $58.6 \mathrm{kcal} \mathrm{mol}^{-1}$, fac-[Ir(Fppy) 3 , resulted in a decreased yield of $61 \%$ for 15 (entry 4, Table 1). Catalysts with triplet energies below $57 \mathrm{kcal} \mathrm{mol}^{-1}$ including $\left[\operatorname{Ir}(\mathrm{dFppy})_{2}(\mathrm{dtbbpy})\right] \mathrm{PF}_{6}$, fac-$_{-}\left[\operatorname{Ir}(\mathrm{ppy})_{3}\right]$, and fac $^{-\left[\operatorname{Ir}\left(4^{\prime}-\mathrm{CF}_{3}-\right.\right.}$

Table 1. Evaluation of photocatalysts in intramolecular, vis-

\begin{tabular}{|c|c|c|c|c|}
\hline$Y_{M}$ & ${\stackrel{N}{N} \lambda_{\mathrm{Me}}^{\mathrm{Me}}}^{-O}$ & $\begin{array}{c}\text { photocatalyst ( } 1 \mathrm{~mol} \% \\
\mathrm{CH}_{3} \mathrm{CN}(0.1 \mathrm{M} \text { ), fan } \\
\text { blue LEDs }\end{array}$ & $\mathrm{Me}$ & $\overbrace{}^{-}\rangle_{\mathrm{Me}}^{\mathrm{Me}}$ \\
\hline entry & photocatalyst & $\mathrm{E}_{\mathrm{T}}\left(\mathrm{kcal} \cdot \mathrm{mol}^{-1}\right)$ & $\lambda(\mathrm{nm})$ & yield $(\%)$ \\
\hline 1 & {$\left[\operatorname{lr}(\mathrm{dF}(\mathrm{Me}) \mathrm{ppy})_{2}(\mathrm{dtbbpy})\right] \mathrm{PF}_{6}$} & 60.2 & 427 & 73 \\
\hline 2 & $\operatorname{Ir} 1 \cdot P F_{6}$ & 60.1 & 427 & 78 \\
\hline 3 & Ir2 & 60.1 & 427 & 90 \\
\hline 4 & $f a c-\left[\operatorname{Ir}(\mathrm{Fppy})_{3}\right]$ & 58.6 & 427 & 61 \\
\hline 5 & {$\left[\operatorname{lr}(\mathrm{dFppy})_{2}(\mathrm{dtbbpy})\right] \mathrm{PF}_{6}$} & 56.4 & 427 & 29 \\
\hline 6 & $f a c-\left[\operatorname{lr}(p p y)_{3}\right]$ & 55.4 & 427 & 3 \\
\hline 7 & fac-[Ir $\left.\left(4^{\prime}-\mathrm{CF}_{3}-\mathrm{ppy}\right)_{3}\right]$ & 55.2 & 427 & 7 \\
\hline 8 & Thioxanthone & 65.5 & 427 & 0 \\
\hline 9 & 2-MeOTx & 57.8 & 427 & 41 \\
\hline 10 & $2 \mathrm{CzPN}$ & 60.6 & 427 & 41 \\
\hline 11 & 4CZIPN & 58.3 & 427 & 0 \\
\hline 12 & Thioxanthone ${ }^{a}$ & 65.5 & 427 & 6 \\
\hline 13 & 2-MeOTx ${ }^{a}$ & 57.8 & 427 & 26 \\
\hline 14 & $2 \mathrm{CzPN}^{\mathrm{a}}$ & 60.6 & 427 & 38 \\
\hline 15 & $4 \mathrm{CzPIN}{ }^{\mathrm{a}}$ & 58.3 & 427 & 19 \\
\hline 16 & Ir2 (0.2 M) & 60.1 & 427 & 76 \\
\hline 17 & Ir2 (0.1 M) & 60.1 & 427 & 84 \\
\hline 18 & Ir2 (0.05 M) & 60.1 & 427 & 85 \\
\hline 19 & Ir2 (0.01 M) & 60.1 & 427 & 85 \\
\hline 20 & no catalyst & - & - & 0 \\
\hline
\end{tabular}
ible light-mediated aza Paternò-Büchi reactions.

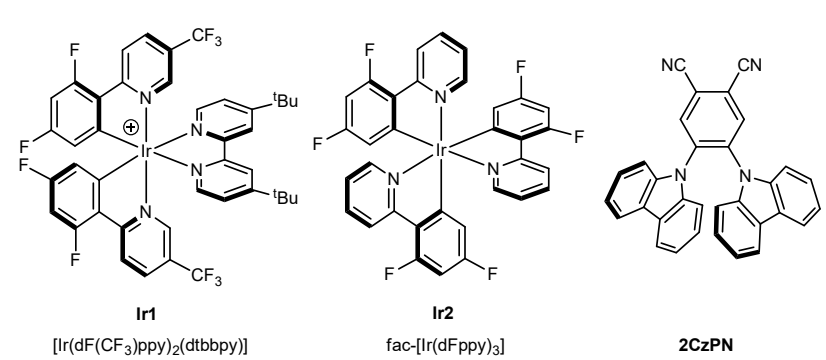

Conditions: $0.1 \mathrm{mmol} 22$ and photocatalyst are dissolved in acetonitrile $(0.1 \mathrm{M})$ and the solution was sparged for $10 \mathrm{~min}$. The sample was irradiated with blue LED lamps 16 hours. Percent yield was determined by NMR with mesitylene as internal

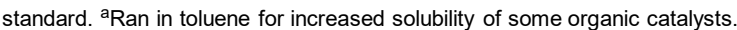


ppy)3] resulted in diminished formation of azetidine 15 in yields of less than 29\% (entries 5-6, Table 1). Organic photocatalysts were also considered under similar conditions, varying the triplet energy of the catalyst used. In particular, utilizing thioxanthone with a higher triplet energy than the optimal iridium catalyst, Ir2, no product was observed (entry 8 , Table 1 ).

Using organic catalysts with lower triplet energies than thioxanthone, 2-MeOTx and $2 \mathrm{CzPN}$, gave rise to azetidine 15 in $41 \%$ yield for both catalysts (entries 9-10, Table 1). However, conversion of oxime 14 relying on 4 CzIPN exhibiting a triplet energy between 2-MeOTx and 2CzPN failed to provide the desired azetidine 15 (entry 12, Table 1). Suspecting that the solubility of the organic photocatalyst may be influencing the yield, the solvent was switched to toluene. Under these modified reaction conditions, thioxanthone produced trace amounts of azetidine 15 in 6\% yield (entry 12, Table 1). 2-MeOTx did not perform as well in toluene as it did in acetonitrile, resulting in a decreased yield of $26 \%$, while 2CzPN performed similarly, producing 15 in 38\% yield (entries 13-14, Table 1). 4CzIPN in toluene produced an increased yield of $19 \%$ (entry 15, Table 1). The optimal organic catalyst conditions were identified as 2-MeOTx or $2 \mathrm{CzPN}$ in acetonitrile, which is the first instance of organic catalysts being used in a visible light-mediated [2+2] cycloaddition between alkenes and oximes to access azetidines products by exciting the oxime to its triplet state.

With an optimal photocatalyst identified, we subsequently focused on further improving the reaction conditions, with a particular emphasis on concentration (Table 2). Specifically, at $0.2 \mathrm{M}$ a conversion of $98 \%$ was observed, however the desired product $\mathbf{1 5}$ was formed in diminished yields of only $76 \%$ (entry 16 , Table 1 ). In comparison, decreasing the concentration to $0.1 \mathrm{M}$ resulted in the formation of azetidine 15 with improved yields of $84 \%$ while complete consumption of oxime 14 was observed (entry 17, Table 1). Notably, did not result in an improvement in yield (entries 18-19, Table 1). Notably, without photocatalyst no formation of azetidine 15 was observed when oximes 14 was irradiated with blue LED light (entry 20, Table 1).

Utilizing the optimized conditions, the substrate scope for intramolecular aza Paternò-Büchi reactions of oximes incorporating unactivated alkenes was explored. The optimal reaction conditions identified proved to be compatible with a variety of alkene substitution patterns, chain lengths, and oxime backbone substituents, providing access to azetidines in up to $87 \%$ yield. The trisubstituted alkene substrate resulted in the formation of tricyclic azetidine $\mathbf{1 5}$ in $87 \%$ yield, while disubstituted alkenes $(19,20$, and 23$)$ produced yields up to $68 \%$ yield. The optimal reaction conditions were found to tolerate terminal alkenes, which was surprising as the postulated reaction mechanism for this intramolecular aza Paternò-Büchi reaction relied on the formation of a primary radical. Employing substrates incorporating terminal alkenes allows access to azetidines with methylene groups adjacent to the nitrogen, which were previously only accessible using ethylene gas following the former intermolecular reaction protocol. Terminal alkene $\mathbf{1 6}$ resulted in a yield of $25 \%$, however this was improved by decreasing the concentration of the reaction to $80 \%$. Substitution adjacent to the ester moiety resulted in a higher yield of $69 \%$ for the dimethyl substrate $\mathbf{1 7}$ although a decrease in yield to $9 \%$ was observed for azetidine 18, bearing a monomethyl substituent. Conversely, the crotyl ester resulted in a $68 \%$ yield of azetidine 19 with a 1:1 mixture of diastereomers. Increasing the size of the substituent to a $n$-propyl group did not improve the diastereoselectivity, however did produce 20 in 61\% yield. Importantly, azetidines incorporating 6-membered ring-lactones were similarly readily accessible, albeit in slightly diminished yields compared to their 5-membered ring analogs. The substrate utilizing a terminal alkene resulted in $\mathbf{2 1}$ being formed in $6 \%$ while the trisubstituted alkene resulted in $\mathbf{2 2}$ being formed in an in-

Table 2. Evaluation of the scope of intramolecular, visible light-mediated aza Paternò-Büchi reactions.

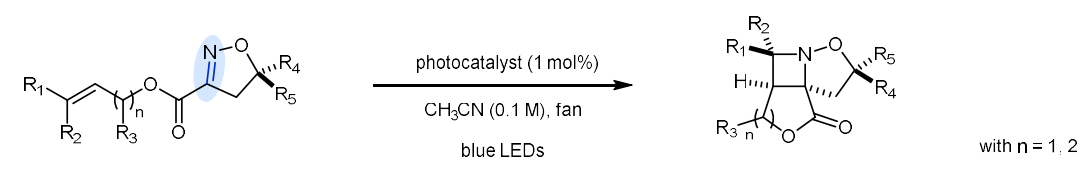

(6)
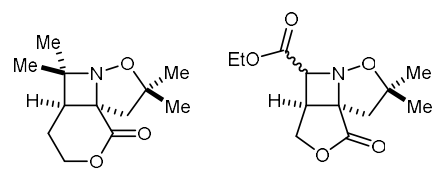

$22\left(41 \%, 71 \%{ }^{a}\right)$

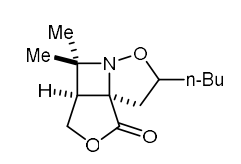

$24(72 \%)$

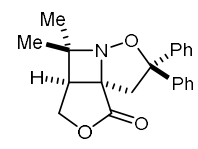

$25\left(22 \%, 61 \%^{a}\right)$

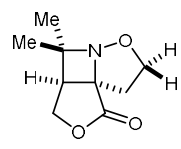

$26(80 \%)$

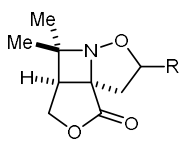

$27(\mathrm{R}=\mathrm{Ph} 58 \%)$

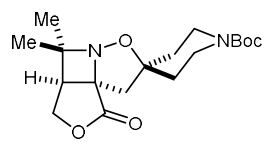

$29(57 \%)$

Conditions: $\quad 0.25 \mathrm{mmol}$ isoxazoline and $\operatorname{Ir} 2(1 \mathrm{~mol} \%)$ are dissolved in acetonitrile $(0.1 \mathrm{M})$ and the solution is sparged for $10 \mathrm{~min}$. The reaction is irradiated with blue LED lamps $(427 \mathrm{~nm})$ for $16-20$ hours. ${ }^{a}$ Reaction run at $0.01 \mathrm{M}$ on $0.05 \mathrm{mmol}$ scale. yield by HNMR.

decreasing the concentration further to $0.05 \mathrm{M}$ or $0.01 \mathrm{M}$ 
creased $41 \%$ due to the increased substitution. By decreasing the reaction concentration for $\mathbf{2 1}$ and 22, the yields increased to $25 \%$ and $71 \%$, respectively. An acrylate was also tolerated under these optimal reaction conditions, resulting in $27 \%$ yield of 23 . Different substitution patterns on the oxime backbone were amenable, including the unsubstituted oxime $\mathbf{2 6}$ resulting in $80 \%$ yield, which is comparable to the $87 \%$ yield of $\mathbf{1 5}$ obtained from the corresponding dimethyl substituted oxime. Monosubstituted oximes were tolerated, however yields varied based on the substituent. Specifically, the $n$-butyl substituent azetidine 24 was produced in $72 \%$ yield, the highest yield for monosubstituted substrates while phenyl (27) and ethyl ether (28) resulted in $58 \%$ and $28 \%$ yields, respectively. Bulkier disubstituted oxime substituents, specifically diphenyl 25 and piperidine 29 , resulted in $22 \%$ and $57 \%$ yield respectively. Dilution of the reaction for 25 to $0.01 \mathrm{M}$ resulted in an improved yield of $61 \%$. Diluting the reaction for select low yielding substrates resulted in the yields improving, importantly, the increasing of the yield for terminal alkene 17, demonstrates that terminal alkenes can work as well as the trisubstituted alkenes under more dilute conditions.

Herein, we report the development of an intramolecular visible light-enabled $[2+2]$-cycloaddition reaction between unactivated alkenes and cyclic oximes to access tricyclic azetidines in yields up to $87 \%$. This transformation relies on an iridium photocatalyst but can similarly be promoted by an organic photocatalyst to achieve yields of the desired product of up to $41 \%$, resulting in complex, highly saturated scaffolds. Notably, this method gives rise to a reverse substitution pattern in comparison to previous methods developed, ${ }^{16,17}$ allowing access to molecules that occupy distinct chemical space. We anticipate this new strategy to be used for the rapid build-up of molecular complexity when incorporating functionalized azetidines in current drug design and development.

\section{ASSOCIATED CONTENT}

Supporting Information. The Supporting Information is available free of charge on the ACS Publications website and includes experimental procedures, characterization $\left({ }^{1} \mathrm{H}-\mathrm{NMR}\right.$, ${ }^{13} \mathrm{C}-\mathrm{NMR}$, IR, and MS data), additional optimization and control experiments (pdf format).

\section{AUTHOR INFORMATION}

\section{Corresponding Author}

* Email: corinnas@umich.edu.

\section{Author Contributions}

The manuscript was written through contributions of all authors. All authors have given approval to the final version of the manuscript.

Notes

The authors declare no competing financial interests.

\section{ACKNOWLEDGMENT}

We thank the Alfred P. Sloan Foundation, the David and Lucile Packard Foundation the Camille and Henry Dreyfus Foundation, and NIH (R01-GM141340) for funding. D.E.B. thanks Emily R. Wearing and Katie A. Rykaczewski for many helpful discussions.

\section{REFERENCES}

(1) Lovering, F.; Bikker, J.; Humblet, C., Escape from flatland: increasing saturation as an approach to improving clinical success. J. Med. Chem. 2009, 52, 6752-6756. https://doi.org/10.1021/jm901241e.

(2) Lovering, F., Escape from Flatland 2: complexity and promiscuity. Med. Chem. Commun. 2013, 4, 515-519. https://doi.org/10.1039/C2MD20347B.

(3) Bauer, M. R.; Di Fruscia, P.; Lucas, S. C. C.; Michaelides, I. N.; Nelson, J. E.; Storer, R. I.; Whitehurst, B. C., Put a ring on it: application of small aliphatic rings in medicinal chemistry. RSC Med. Chem. 2021, 12, 448-471. https://doi.org/10.1039/D0MD00370K.

(4) Fish, P. V.; Brown, A. D.; Evrard, E.; Roberts, L. R., 7Sulfonamido-3-benzazepines as potent and selective 5-HT2C receptor agonists: hit-to-lead optimization. Bioorg. Med. Chem. $\begin{array}{llll}\text { Lett. } & \mathbf{2 0 0 9}, & 19, & 1871-5 .\end{array}$ https://doi.org/10.1061/j.bmcl.2009.02.071.

(5) St Jean, D. J., Jr.; Fotsch, C., Mitigating heterocycle metabolism in drug discovery. J. Med. Chem. 2012, 55, 60026020. https://doi.org/10.1021/jm300343m.

(6) Shu, Y. Z.; Johnson, B. M.; Yang, T. J., Role of biotransformation studies in minimizing metabolism-related liabilities in drug discovery. AAPS J. 2008, 10, 178-192. https://doi.org/10.1208/s12248-008-9016-9.

(7) Lowe, J. T.; Lee, M. D. t.; Akella, L. B.; Davoine, E.; Donckele, E. J.; Durak, L.; Duvall, J. R.; Gerard, B.; Holson, E. B.; Joliton, A.; Kesavan, S.; Lemercier, B. C.; Liu, H.; Marie, J. C.; Mulrooney, C. A.; Muncipinto, G.; Welzel-O'Shea, M.; Panko, L. M.; Rowley, A.; Suh, B. C.; Thomas, M.; Wagner, F. F.; Wei, J.; Foley, M. A.; Marcaurelle, L. A., Synthesis and profiling of a diverse collection of azetidine-based scaffolds for the development of CNSfocused lead-like libraries. J. Org. Chem. 2012, 77, 7187-7211. https://doi.org/10.1021/jo300974j.

(8) Brown, A.; Brown, T. B.; Calabrese, A.; Ellis, D.; Puhalo, N.; Ralph, M.; Watson, L., Triazole oxytocin antagonists: Identification of an aryloxyazetidine replacement for a biaryl substituent. Bioorg. Med. Chem. Lett. 2010, 20, 516-520. https://doi.org/10.1016/bmcl.2009.11.097.

(9) Maetani, M.; Zoller, J.; Melillo, B.; Verho, O.; Kato, N.; Pu, J.; Comer, E.; Schreiber, S. L., Synthesis of a Bicyclic Azetidine with In Vivo Antimalarial Activity Enabled by Stereospecific, Directed C(sp(3))-H Arylation. J. Am. Chem. Soc. 2017, 139, 11300-11306. https://doi.org/10.1021/jacs.7b06994.

(10) Vitaku, E.; Smith, D. T.; Njardarson, J. T., Analysis of the structural diversity, substitution patterns, and frequency of nitrogen heterocycles among U.S. FDA approved pharmaceuticals. J. Med. Chem. 2014, 57, 10257-10274. https://doi.org/10.1021/im501100b.

(11) Cromwell, N. H.; Phillips, B., The Azetidines. Recent Synthetic Developments. Chem. Rev. 1979, 79, 331-358. https://doi.org/10.1021/cr60320a003.

(12) Richardson, A. D.; Becker, M. R.; Schindler, C. S., Synthesis of azetidines by aza Paterno-Buchi reactions. Chem. Sci. 2020, 11, 7553-7561. https://doi.org/10.1039/D0SC01017K.

(13) Brandi, A.; Cicchi, S.; Cordero, F. M., Novel syntheses of azetidines and azetidinones. Chem. Rev. 2008, 108, 3988-4035. https://doi.org/10.1021/cr800325e.

(14) Antermite, D.; Degennaro, L.; Luisi, R., Recent advances in the chemistry of metallated azetidines. Org. Biomol. Chem. 2016, 15, 34-50. https://doi.org/10.1039/C60b01665K. 
(15) Gianatassio, R.; Lopchuk, J. M.; Wang, J.; Pan, C. M.; Malins, L. R.; Prieto, L.; Brandt, T. A.; Collins, M. R.; Gallego, G. M.; Sach, N. W.; Spangler, J. E.; Zhu, H.; Zhu, J.; Baran, P. S., Organic chemistry. Strain-release amination. Science 2016, 351, 241246. https://doi.org/10.1126/science.aad6252.

(16) Becker, M. R.; Richardson, A. D.; Schindler, C. S., Functionalized azetidines via visible light-enabled aza PaternoBuchi reactions. Nat. Commun. 2019, 10, 5095. https://doi.org/10.1038/s41467-019-13072-x.

(17) Becker, M. R.; Wearing, E. R.; Schindler, C. S., Synthesis of azetidines via visible-light-mediated intermolecular [2+2] photocycloadditions. Nat. Chem. 2020, 12, 898-905. https://doi.org/10.1038/s41557-020-0541-1.

(18) Wearing, E. R.; Blackmun, D.E.; Schindler, C. S., 1- and 2Azetines via Visible Light-Mediated [2+2]-Cycloadditions of Alkynes and Oximes. J. Am. Chem. Soc. 2021, 143, 16235-16242. https://doi.org/10.1021/jacs.1c07523.

(19) Rykaczewski, K.A.; Schindler, C. S. Visible-Light-Enabled Paternò-Büchi Reaction via Triplet Energy Transfer for the Synthesis of Oxetanes. Org. Lett. 2020, 22, 6516-6519. https://doi.org/10.1021/acsorglett.0c02316. 